\title{
Stochastic resonance quantified by statistical complexity measures in a bistable system subject to colored noise
}

\author{
Meijuan He • Wei Xu • Zhongkui Sun • Wantao Jia
}

Received: 7 March 2013 / Revised: 12 July 2013 / Accepted: 22 July 2013 / Published online: 3 August 2013

(C) Springer-Verlag Berlin Heidelberg 2013

\begin{abstract}
This work deals mainly with the problem of the stochastic resonance in a symmetric bistable system subject to colored noise by virtue of the statistical complexity measures. Firstly, based on Bandt-Pompe methodology, the statistical complexity measures are dedicated to quantify the stochastic resonance of the system. Then, the effects of the correlation time of the colored noise, the amplitude and the frequency of the signal on the stochastic resonance are discussed by using the statistical complexity measures. Meanwhile, the SNR is also given for the purpose of verifying the validity of this approach. It is turned out that the statistical complexity measures can be used to characterize some subtle signatures of noise-induced phenomena. Additionally, the robustness of the results is verified with reducing the embedding dimension and the total length of the time series.
\end{abstract}

Keywords Stochastic resonance .

Statistical complexity measures · Colored noise ·

Signal-to-noise ratio

\section{Introduction}

Stochastic resonance was originally proposed by Benzi and his coworkers in 1981 to interpret the periodic recurrences of the earth's ice ages [1], which has attracted lots of attention now and got its potential application in a wide range, for instance, in paleoclimatology [2], electronic circuits [3], lasers [4], chemical systems [5], biological systems [6] and so on. Consequently, great efforts have been devoted to it and many results have been reported [7-19]. To quantify the

M. He $(\bowtie) \cdot$ W. Xu $\cdot$ Z. Sun $\cdot$ W. Jia

Department of Applied Mathematics, Northwestern Polytechnical

University, Xi' an 710072, People's Republic of China

e-mail: meijuan@mail.nwpu.edu.cn onset of stochastic resonance, several approaches (or indicators) have been suggested, such as signal-to-noise ratio (SNR), Fokker-Planck description, linear-response theory and residence-time distributions. Among these approaches, undoubtedly, SNR is the most popular one, which indicates the ratio of the $\delta$ peak height in the power spectrum to the noise background. The expression of SNR was firstly derived by McNamara et al. [8] in the adiabatic limit through considering a master equation of two-state model. And it was extensively adopted by many authors in their papers and reviews $[14,17,18]$. However, in order to obtain the representation of SNR and model the nonadiabatic regime of a given dynamical system, it is essential to calculate the corresponding Fokker-Planck equation and probability density. Hence, Fokker-Planck equation plays a prominent role in the description of stochastic resonance, and has been repeatedly invoked and investigated $[17,18]$. Moreover, another elaborate criterion of the stochastic resonance phenomenon is the linear-response theory, which was proposed by Dykman et al. [11-13] to investigate an overdamped bistable system under a weak periodic force and an external noise. In particular, this method is also applied within the framework of nonstationary stochastic processes. Recently, a new and feasible tool, that is, statistical complexity measures for quantifying stochastic resonance was proposed by Rosso et al. $[15,16]$ when they studied a Brownian particle in a sinusoidally modulated bistable potential.

Statistical complexity measure is a functional to characterize the probability distribution associated with the time series generated by a dynamical system. Therefore, it not only verifies the randomness but also describes the correlation structures of a given system. In recent years, a vast majority of research on the measures of complexity have been extensively done [20-26]. López-Ruiz et al. first proposed a definition of statistical complexity measure based on a 
probabilistic description of physical systems, and discovered accessible states and probability distribution of the dynamical evolution of a given system by this scheme [20]. Then, Martin et al. studied the concept of disequilibrium as an essential ingredient of statistical complexity measures, and replaced the Euclidean distance by the Wootter' distance and the Jensen-Shannon divergence, respectively. Meanwhile, they applied the "cured" complexity measures to the logistic map and clearly exhibited its advantages [21,22]. In 2007, Rosso et al. introduced a representation space called the Complexity- Entropy causality plane, and showed that it was a useful method for dealing with the subtle difference between noise and chaos [23]. Subsequently, Zunino et al. revealed the existence of a time delay by using the permutation entropy and the permutation statistical complexity in the standard well-known Mackey-Glass system, and demonstrated that the permutation approach was helpful for unveiling the presence of a time delay in time delay identification scenarios [26].

With the previous descriptions and studies [15,16], it was shown that, in the case of Gaussian white noise, the entropy displayed a minimum and the complexity measure exhibited a maximum for an optimal level of noise, which were regarded as the feature of resonant-like behavior. It is also found that statistical complexity measures had great potential for the precise detection of subtle signatures under analysis. However, to our knowledge, the effect of colored noise on stochastic resonance by means of complexity measures has not been reported yet. Motivated by the above discussions, in this paper, we focus on the statistical complexity measures which are used to detect the constructive role of colored noise in a bistable system assisted by a periodic signal.

This paper is organized as follows. In Sect. 2, Langevin equation under additive colored noise is introduced, and the SNR is calculated. And then, in Sect. 3, the definition of the statistical complexity measures are described in detail. Later, the phenomenon of stochastic resonance together with statistical complexity measures is investigated in Sect. 4. Finally, the conclusions are proposed in Sect. 5.

\section{Model and SNR}

A Langevin equation with additive colored noise in the presence of a periodic signal is governed by

$\dot{x}=-U_{0}^{\prime}(x)+A \cos (\omega t)+\xi(t)$,

where the symmetric double-well potential $U_{0}(x)=-x^{2} / 2$ $+x^{4} / 4$ has two stable states $x_{s 1}=1, x_{s 2}=-1$ and an unstable state $x_{u n}=0 ; A$ and $\omega$ represent the amplitude and the frequency of the periodic signal, respectively; $\xi(t)$ is the Ornstein-Uhlenbek noise with intensity $Q$ and correlation time $\tau$, satisfied statistical properties

$$
\begin{aligned}
<\xi(t)> & =0, \\
<\xi(t) \xi(s)> & =\frac{Q}{\tau} \exp \left(-\frac{|t-s|}{\tau}\right) .
\end{aligned}
$$

Due to the non-Markovian nature of nonlinear system subjected to Gaussian colored noise, it is very hard to obtain approximate Fokker-Planck equation. So many approximate methods have been proposed, for example, decoupling ansatz [27,28], unified colored noise approximation (UCNA) [29-31], etc. Set $\tilde{U}(x)$ to be the generalized potential, and it can be given as

$$
\begin{aligned}
\tilde{U}(x)= & \frac{\tau}{2} x^{6}+\left(\frac{1}{4}-\tau\right) x^{4}-\tau A \cos (\omega t) x^{3} \\
& +\frac{\tau-1}{2} x^{2}+(\tau-1) A \cos (\omega t) x \\
& +\frac{D}{2} \ln D-D \ln \left[1-\tau\left(1-3 x^{2}\right)\right] .
\end{aligned}
$$

According to Ref. [32], the transition rates $W_{ \pm}$can be derived by using steepest descent method, which has the following form

$$
\begin{aligned}
W_{ \pm}= & \frac{\left[\left|U_{0}^{\prime \prime}\left(x_{u n}\right) U_{0}^{\prime \prime}\left(x_{s-}\right)\right|\right]^{\frac{1}{2}}}{2 \pi} \exp \\
& \times\left\{-\frac{1}{Q}\left[\tilde{U}\left(x_{u n}\right)-\tilde{U}\left(x_{s-}\right)\right]\right\} .
\end{aligned}
$$

Therefore, the SNR can be determined from (4) as

$S N R=\frac{\pi \alpha_{1}^{2} A^{2}}{4 \alpha_{0}}\left[1-\frac{\alpha_{1}^{2} A^{2}}{2\left(\alpha_{0}^{2}+\omega^{2}\right)}\right]^{-1}$,

with $\beta=A \cos (\omega t), \frac{1}{2} \alpha_{0}=\left.W_{ \pm}\right|_{\beta=0}, \frac{1}{2} \alpha_{1}=-\left.\frac{d W_{ \pm}}{d \beta}\right|_{\beta=0}$. By virtue of this expression, the stochastic resonance phenomenon of the Eq. (1) has been widely studied by amounts of researchers over the past decades, and many perfect results were reported in their papers. In the present paper, we intend to use a new tool, statistical complexity measures, again to quantify the stochastic resonance of this system.

\section{Statistical complexity measures}

An information measure can be regarded as a quantity describing a given probability distribution. Shannon's logarithmic information measure is defined by the form $S[P]=-\sum_{j=1}^{N} p_{j} \ln \left(p_{j}\right)$, which denotes the uncertainty of the physical process with the probability distribution $P=\left\{p_{j}, j=1, \ldots, N\right\}$. Obviously, in the case of $S[P]=0$, the underlying system characterized by the probability distribution $P$ reaches an extreme circumstance of "perfect order". Otherwise, the given process has a maximum "randomness" if $S[P]=\ln N$. Based on the given probability distribution 
$P$ and the corresponding information measure $S[P]$, the definition of "disorder" $\mathcal{H}$, i.e., the normalized Shannon entropy is given as

$\mathcal{H}[P]=S[P] / S_{\max }$,

where $S_{\max }=S\left[P_{e}\right]=\ln N$ and $P_{e}=\{1 / N, \ldots, 1 / N\}$ is the uniform distribution. Then $0 \leq \mathcal{H}[P] \leq 1$.

It follows that statistical complexity measure cannot be defined in terms of just "disorder" or "information". It seems more reasonable to propose a measure of "statistical complexity" by adopting some kind of distance $\mathcal{D}$ to the uniform distribution $P_{e}$ [20-22,24]. For this purpose, one can define the "disequilibrium" as

$\mathcal{Q}[P]=Q_{0} \cdot \mathcal{D}\left[P, P_{e}\right]$,

where $Q_{0}$ is a normalization constant and $0 \leq \mathcal{Q} \leq 1$. It reflects the "architecture" of the system. Here, the distance $\mathcal{D}$ is interpreted and calculated as the Jensen-Shannon divergence $\mathcal{J}_{S}$, which satisfied

$$
\mathcal{J}_{S}\left[P_{1}, P_{2}\right]=\left\{S\left[\left(P_{1}+P_{2}\right) / 2\right]-S\left[P_{1}\right] / 2-S\left[P_{2}\right] / 2\right\} .
$$

Thus the disequilibrium can be further expressed as

$$
\mathcal{Q}_{J}[P]=Q_{0} \cdot \mathcal{J}_{S}\left[P, P_{e}\right] .
$$

Here the index $J$ stands for the Jensen-Shannon divergence of the distance, and the constant $Q_{0}$ is equal to the reciprocal of $\mathcal{J}_{S}\left[P, P_{e}\right]$ when the value of $\mathcal{J}_{S}\left[P, P_{e}\right]$ is maximal, namely,

$Q_{0}=-2\left\{\left(\frac{N+1}{N}\right) \ln (N+1)-2 \ln (2 N)+\ln N\right\}^{-1}$.

Therefore, the following functional form for the statistical complexity measure is adopted:

$\mathcal{C}[P]=\mathcal{Q}_{J}[P] \cdot \mathcal{H}[P]$.

This quantity reflects on not only the interplay between the amount of information stored in the system but also its disequilibrium [20].

From the expression of statistical complexity, it reads immediately that one can calculate the value of statistical complexity iff $\mathcal{H}$ and $\mathcal{Q}_{J}$ were determined. However, it is not an easy job to find an appropriate probability distribution $P$ from the time series $\left\{x_{s}, s=1, \ldots, M\right\}$ of a given system. Several approaches have been suggested as the candidates, among which the Bandt and Pompe (BP) methodology [33] was the widely used one. (See Appendix)

\section{Stochastic resonance}

In this section, we apply the statistical complexity measures as the new measurement to investigate the phenomenon of stochastic resonance of system (1), which is further verified by virtue of the SNR. The Fourth-order Runge-Kutta algorithm is adopted here to solve system (1) numerically. And the Bandt-Pompe method is also employed to calculate the probability distribution from the time series of the consecutive residence time intervals. In addition, the embedding dimension is determined as $D=6$ and the total length is fixed as $M=60,000$ of the analyzed time series.

\subsection{Stochastic resonance}

In this subsection, the stochastic resonance of system (1) is detected with the help of the statistical complexity measures when choosing the parameters as $A=0.10, \omega=0.05$, $\tau=0.01$. The result of the SNR is also performed for the purpose of checking the validity of this method.

The dependence of the statistical complexity measures and the SNR on the noise intensity is illustrated in Fig. 1. First of all, it is seen from Fig. 1a that the curve of the statistical complexity is non-monotonous. With the increment of $Q$, the curve of the complexity increases at first, and then reaches a maximum on a certain noise intensity. Later, this curve presents decreasing when continually increasing the value of $Q$. By virtue of the definition of the statistical complexity, one can see that the degree of the intricate patterns of the bistable system becomes stronger gradually with the increment of the noise intensity, and reaches a maximum level. Subsequently, it will get weaker when $Q$ further increases. Figure 1a shows that the statistical complexity has a maximum on a certain value of the noise intensity which is the symbol of resonantlike behavior. Second, in Fig. 1b, the trend of the normalized Shannon entropy is opposite to the statistical complexity's. The curve of the entropy continuously decreases to a minimum first but gradually increases later. Based on the definition of the normalized Shannon entropy, it is easy to know that, with the increment of the noise intensity, the motion of the bistable system reaches some degree of order, and then this state is destroyed. These phenomena also show that the stochastic resonance occurs. Furthermore, in order to indicate the statistical complexity measures better, the curve of the SNR is given in Fig. 1c. There is a maximum of the SNR curve of the system which is also identified as characteristic of the stochastic resonance phenomenon. By comparing these figures, it is easily found that the results described by the statistical complexity measures are consistent with that quantified by the SNR. Meanwhile, it is also shown in Fig. 1a and $\mathrm{b}$ that the curves of the statistical complexity and the normalized Shannon entropy have some smaller peaks, which indicates that some subtle stochastic resonances exist. Therefore, it follows that the statistical complexity measures could be used to characterize some subtle signatures of noise-induced phenomena. 
Fig. 1 a Complexity, b Entropy and $\mathbf{c}$ SNR as a function of the colored noise intensity $Q$ with $A=0.10, \omega=0.05, \tau=0.01$
Fig. 2 a Complexity, b Entropy and $\mathbf{c}$ SNR as a function of the noise intensity $Q$ for different values of the correlation time $\tau$. $A=0.10, \omega=0.05$
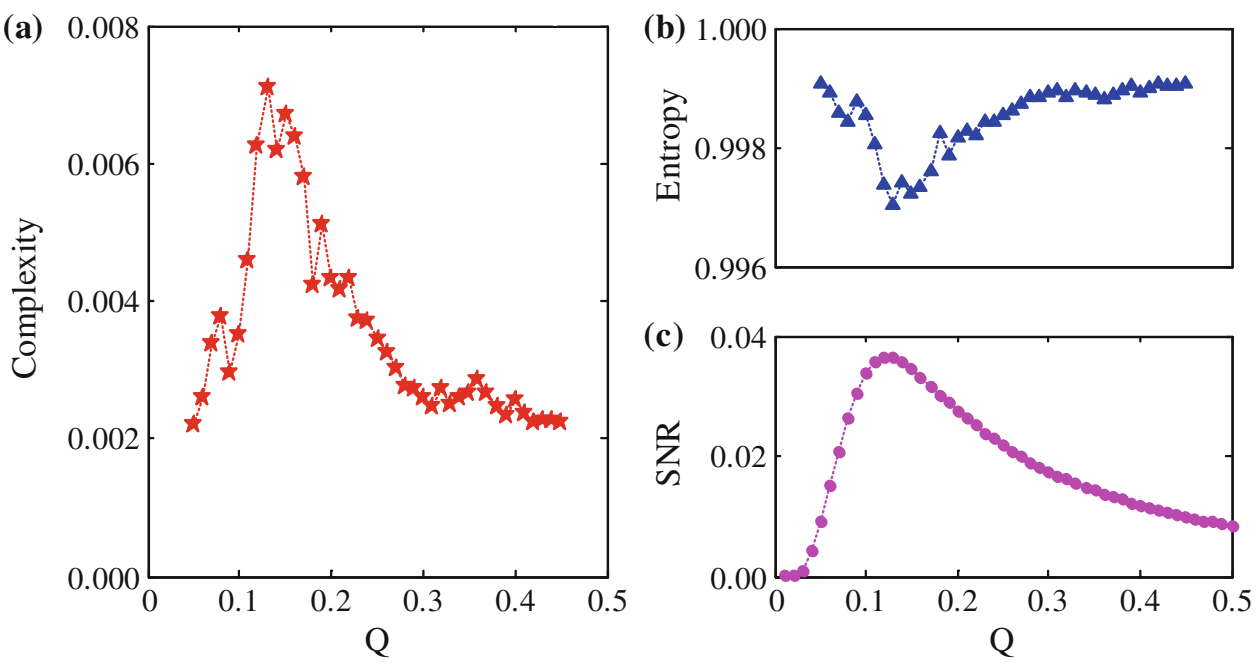
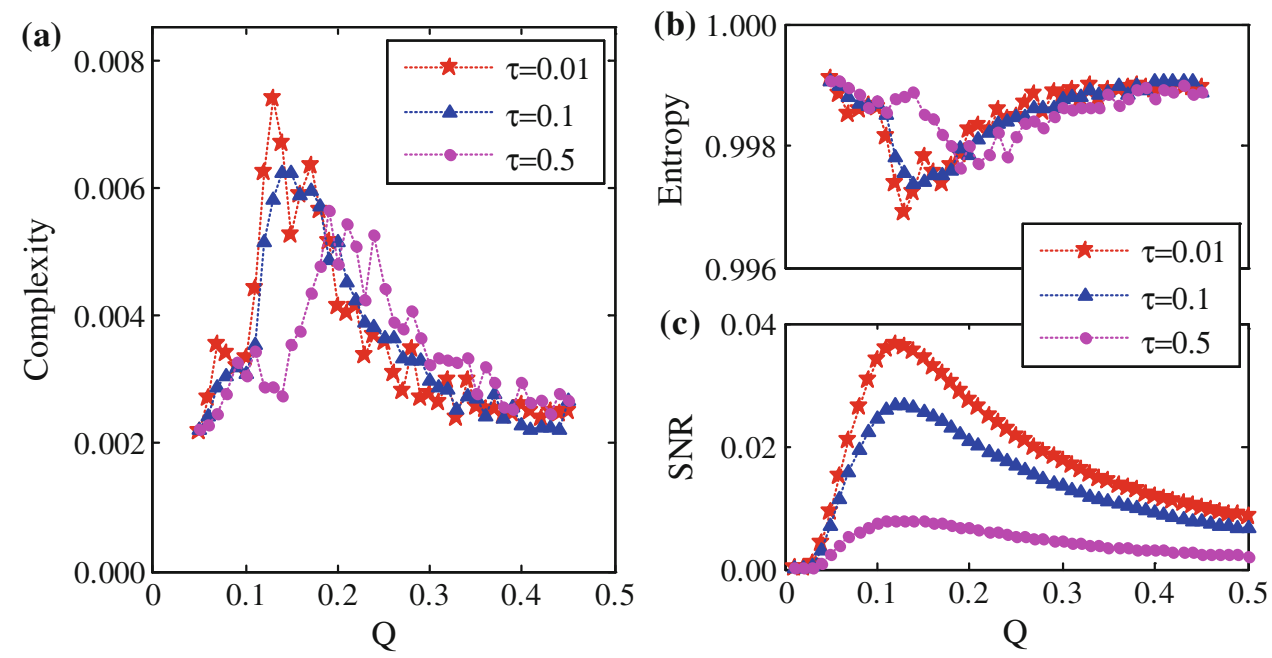

\subsection{Effects of parameters}

Further studies are carried out here to study the effects of the correlation time of the noise, the frequency and the amplitude of the periodic signal on the stochastic resonance of the system by means of the statistical complexity measures. Similarly, these results are compared with the SNR.

The effect of the correlation time $\tau$ of the colored noise on stochastic resonance has been shown in Fig. 2. With the different values of $\tau$, we present the statistical complexity measures as a function of the noise intensity $Q$ in Fig. 2a and $b$. One can see that each curve has an optimum level of noise intensity where the statistical complexity of the system has a maximum and the normalized Shannon entropy has a minimum, which is identified as characters of the occurrence of the stochastic resonance. Furthermore, in Fig. 2a, it is also found that the maximum of the curves of the statistical complexity continuously decreases while increasing the value of $\tau$. And the position of the peak shifts to the right side of the coordinate. Conversely, the peak value of the normalized Shannon entropy gradually increases as increasing the correlation time in Fig. 2b. The peak position also appears the significant deviation towards the right side. It is easy to obtain the conclusion that the phenomenon of stochastic resonance decreases by means of the statistical complexity measures with increasing the correlation time of the noise. To verify the correctness of this result, the curve of the SNR versus the noise intensity $Q$ with varied values of $\tau$ is plotted in Fig. 2c. There exists a single peak in each curve and the stochastic resonance appears. It can also be seen that the SNR decreases with the increment of the correlation time. That is, the stochastic resonance phenomenon is weakened. Therefore, we can show that there is unanimous agreement by comparing the above-mentioned conclusions.

In Fig. 3, the curves of the statistical complexity measures and the SNR, which are considered as a function of the noise intensity $Q$, are described with varied values of the periodic signal frequency $\omega$. On the one hand, one can see from Fig. 3a 
Fig. 3 a Complexity, b Entropy and $\mathbf{c}$ SNR as a function of the noise intensity $Q$ for different values of the periodic signal frequency $\omega$. $A=0.10, \tau=0.01$

Fig. 4 a Complexity, b Entropy and $\mathbf{c}$ SNR as a function of the noise intensity $Q$ for different values of the periodic signal amplitude $A$. $\omega=0.05, \tau=0.01$
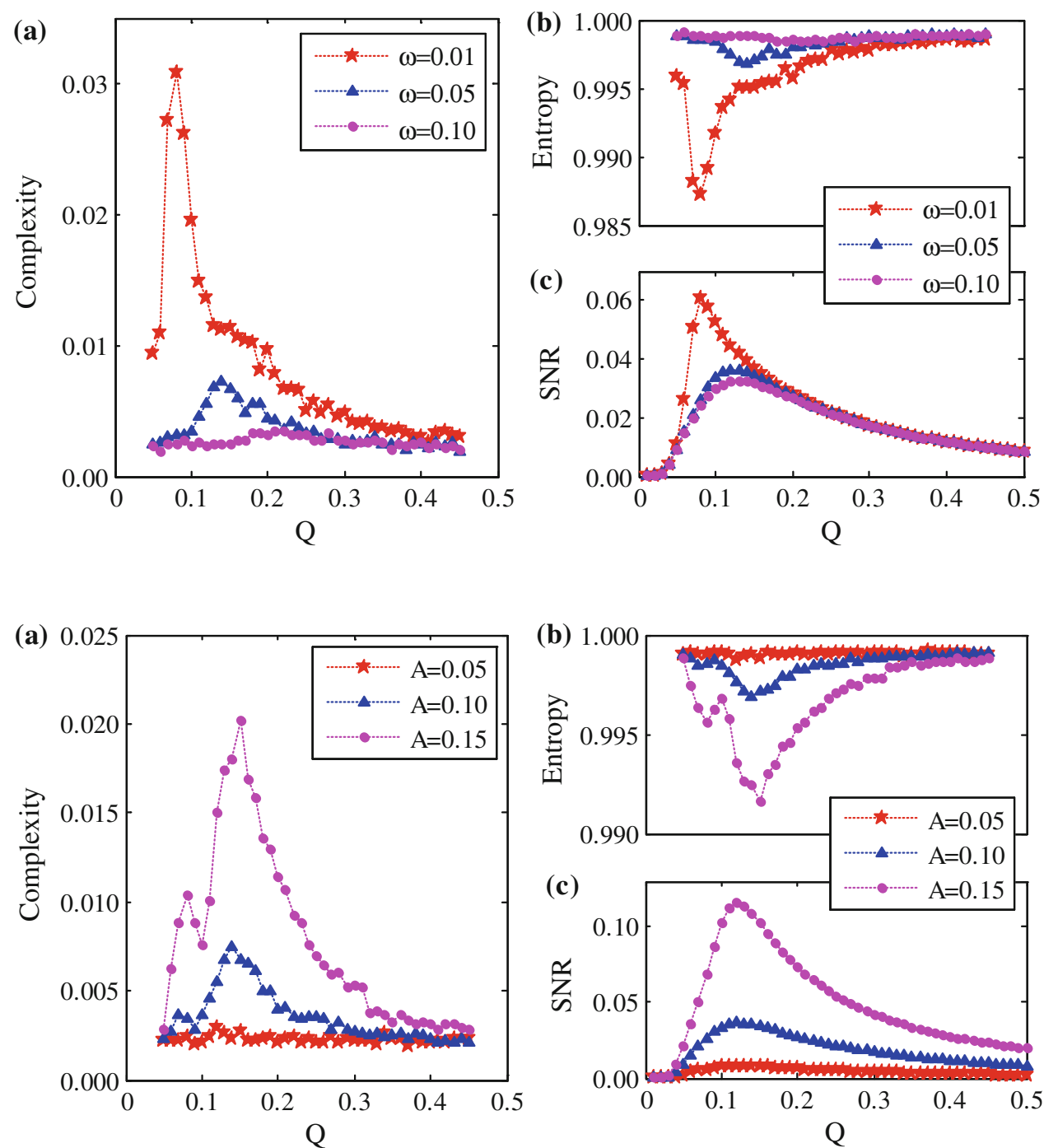

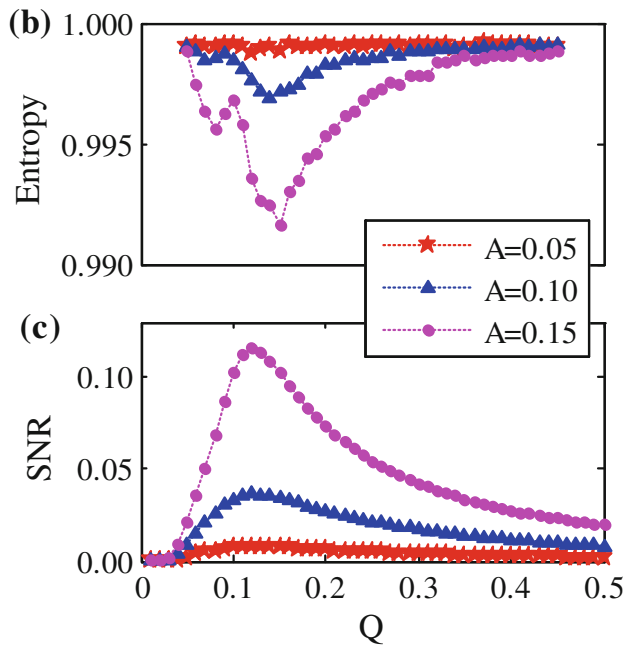

and $b$ that each curve of the statistical complexity exhibits a maximum on a certain noise intensity and the normalized Shannon entropy has a minimum on the same noise intensity, which illustrates that the stochastic resonance phenomenon appears. When increasing the value of $\omega$, the peak value of the statistical complexity continuously decreases first but vanishes later, whereas the minimum of the normalized Shannon entropy increases and then disappears. Moreover, it is also seen that the location of the peaks of the statistical complexity measures shifts to the right side of the axis. These phenomena indicate that the stochastic resonance is suppressed with increasing the signal frequency $\omega$. On the other hand, the curve of the SNR appears a maximum for different $\omega$ in Fig. $3 \mathrm{c}$ which indicates that the stochastic resonance exists. So, we also find that the phenomenon of the stochastic resonance is weakened with the increment of the frequency. According to these three figures, we can also show that the conclusions obtained by the statistical complexity measures are in consonance with that by the SNR.
The results of the statistical complexity measures and the SNR are given in Fig. 4 for different values of the periodic signal amplitude $A$. Obviously, the curves of the statistical complexity measures are non-monotonic and the stochastic resonance appears. When increasing the value of the amplitude, the peak of the statistical complexity increases in Fig. 4a while that of the normalized Shannon entropy decreases in Fig. 4b. It means that the signal amplitude can promote the stochastic resonance phenomenon. In addition, one can also see that the stochastic resonance exists and is strengthened with the increment of the amplitude of the signal in Fig. 4c. But above all, there is also a consistency between the conclusions of the statistical complexity measures and that by the SNR. In particular, there are two peaks of the curves of the statistical complexity measures in the case of $A=0.15$, which implies that the phenomenon of double stochastic resonance happens. But the curve of the SNR still remains one peak for this case. After comparing these two outcomes, we can obtain that the statistical complexity 
Fig. 5 a Complexity and b Entropy as a function of the noise intensity $Q$ for different values of the embedding dimension $D . A=0.10$, $\omega=0.05, \tau=0.01$
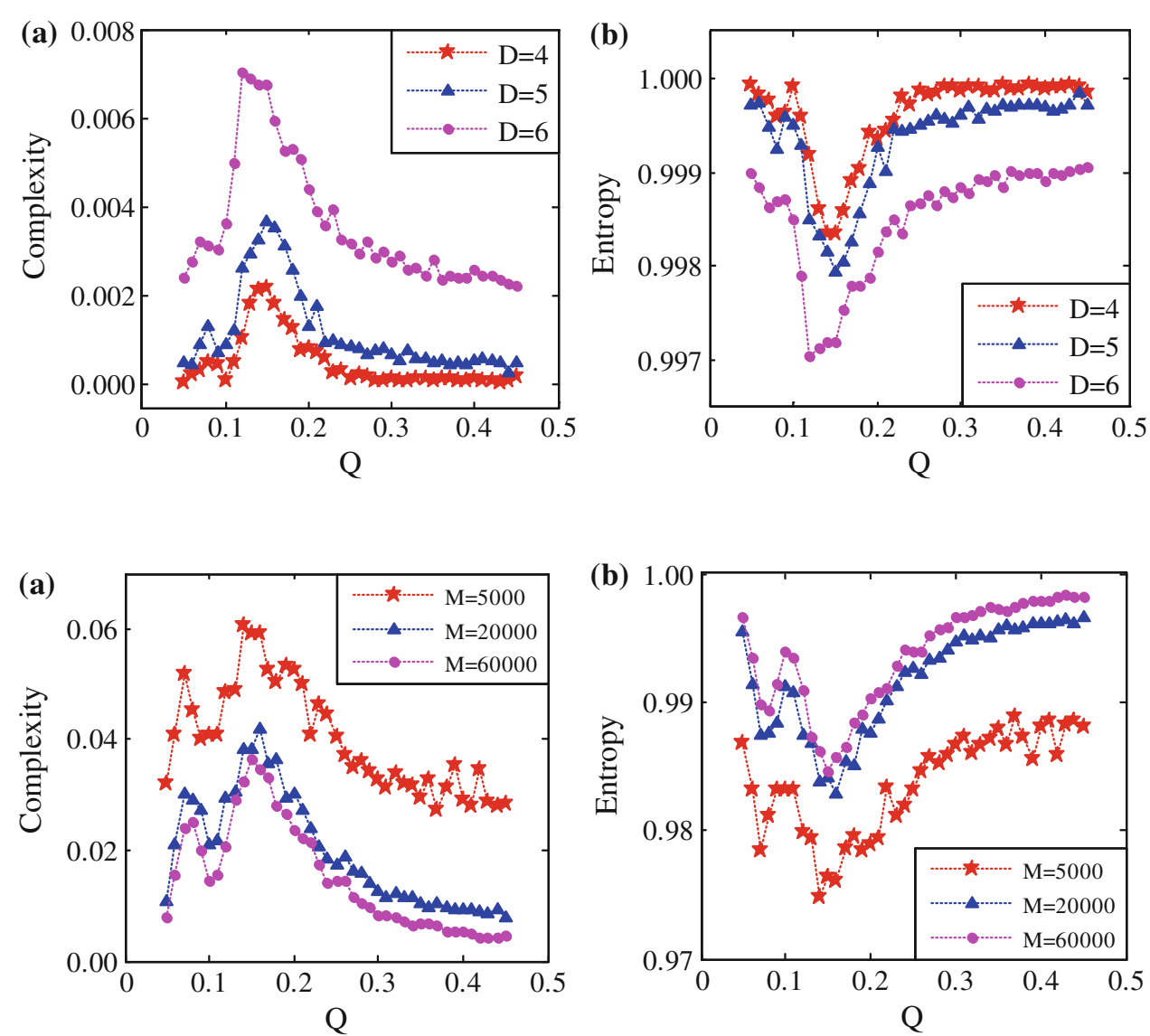

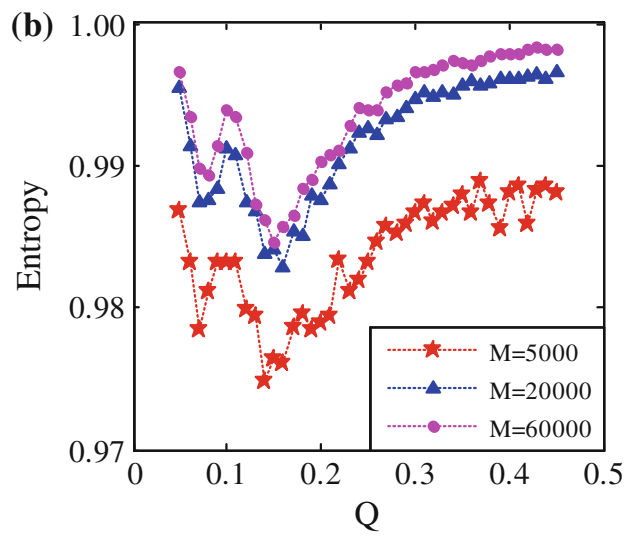

Fig. 6 a Complexity and b Entropy as a function of the noise intensity $Q$ for different values of the total length $M$ of consecutive residence times. $A=0.20, \omega=0.05, \tau=0.01$ measures are more suitable for detecting the stochastic resonance.

\subsection{Robustness}

We discuss the effects of the embedding dimension and the total length of the time series on the robustness of the results in this subsection.

First of all, we study the effect of embedding dimension $D$ on the statistical complexity measures in Fig. 5. It is clear that the values of the complexity measure and the normalized Shannon entropy vary with the embedding dimension $D$. But, the shape of these curves changes slightly in this case. Namely, the result of the occurrence of the stochastic resonance is also found in the case of $D=4$ and $D=5$. In addition, in Fig. 6, the curves of the statistical complexity measures versus the noise intensity $Q$ are given with different values of the total length $M$ of the time series. Similarly to the previous analysis, there doesn't exist drastically change on the shape of the curves and the curves still have two peaks. It is also shown that the stochastic resonance phenomenon occurs with reducing the value of $M$. As summary, these conclusions are consistent with the results on Ref. [15], and demonstrate that the results are robust with respect to the value of $D$ and $M$. This implies that the probability distribu- tion of "ordinal patterns" generated by Bandt-Pompe method is stationary and feasible under the condition of $M \gg D$ !.

\section{Conclusion}

In this paper, the statistical complexity measures have been dedicated to investigate the phenomenon of stochastic resonance in a bistable system driven by an additive colored noise and a periodic signal. First of all, the generalized potential is derived by applying the method of UCNA, and then the expression of the SNR of the system is obtained by means of the two-state approach. What's more, we introduce the definition of the statistical complexity measures, namely, the statistical complexity and the normalized Shannon entropy. Through Fourth-order Runge-Kutta algorithm and BP method, the curves of the statistical complexity measures as a function of the noise intensity are discussed. We can see that the curves of the statistical complexity and the normalized Shannon entropy are non-monotonic, which illustrates the occurrence of the stochastic resonance of the system. In order to check the validity of this method, we also give the result of the SNR and find that there is a consensus between these two conclusions. Meanwhile, we also show that the statistical complexity measures are applied to detect the existence of some subtle behaviors of noise-induced 
phenomena. Furthermore, the statistical complexity measures are used to discuss the effects of the correlation time of the colored noise, the frequency and the amplitude of the periodic signal on stochastic resonance. It can be found that the stochastic resonance is suppressed with the increment of the correlation time and the frequency, but is promoted as increasing the amplitude. These conclusions are also verified by the SNR. In addition, the robustness of the results are shown when the embedding dimension and the total length of time series of successive residence time are reduced, which also demonstrates that the probability distribution of "ordinal patterns" obtained by BP algorithm is stationary.

Acknowledgments This work is supported by the National Nature Science Foundation of China (Grants No. 11172233, No. 10932009, No. 11272258, and No. 11002110) and the NPU Foundation for Fundamental Research (Grant No. JC20110281).

\section{Appendix}

At present, there are three common methods to extract the probability distribution from underlying time series: (a) histograms of the $x_{i}$-vallues; (b) binary representations; (c) BP methodology. In Ref. [23], BP algorithm was regarded as a suitable tool to obtain the probability distribution by comparing with the above three approaches, because it taken into account the time causality of the underlying system better. The detailed description of the BP method is as follows:

Given a one-dimensional time series $\left\{x_{s}: s=1, \ldots, M\right\}$ with embedding dimension $D>1$, the "ordinal pattern" of order $D$ generated by

$(s) \rightarrow\left(x_{s-(D-1)}, x_{s-(D-2)}, \ldots, x_{s-1}, x_{s}\right)$

is considered. To each time $s$, a $D$-dimensional vector from the given time series at times $s-(D-1), \ldots, s-1, s$ is assigned. By the "ordinal pattern" of order $D$ depended on the time $s$, the permutation $\pi=\left(r_{0}, r_{1}, \ldots, r_{D-1}\right)$ of $(0,1, \ldots, D-1)$ is defined as

$x_{S-r_{D-1}} \leq x_{S-r_{D-2}} \leq \cdots \leq x_{S-r_{1}} \leq x_{s-r_{0}}$.

In order to obtain a unique result, BP considered that $r_{i}<$ $r_{i-1}$ if $x_{s-r_{i}}=x_{s-r_{i-1}}$. Thus, for all $D$ ! possible permutations $\pi$ of order $D$, the corresponding probability distribution of "ordinal patterns" is expressed by

$p(\pi)=\#\{s: s \leq \mathcal{Y} ;(s)$ has type $\pi\} / \mathcal{Y}$

where $\mathcal{Y}=M-D+1$, and the symbol \# represents "number".

Since embedding dimension $D$ determines the number of accessible states $D$ ! of the system, for practical purposes, Bandt and Pompe recommended to choose $3 \leq D \leq 7$. In addition, the total lengths $M$ of the time series must satisfy the condition $M \geq D$ ! for the purpose of obtaining a reliable statistics and proper distinction between stochastic and deterministic dynamics.

\section{References}

1. Benzi R, Sutera A, Vulpiani A (1981) The mechanism of stochastic resonance. J Phys A: Math Gen 14:L453-L457

2. Nicolis C (1982) Stochastic aspects of climatic transitions-response to a periodic forcing. Tellus 34:1-9

3. Fauve S, Heslot F (1983) Stochastic resonance in a bistable system. Phys Lett A 97:5-7

4. Iannelli JM, Yariv A, Chen TR, Zhuang YH (1994) Stochastic resonance in a semiconductor distributed-feedback laser. Appl Phys Lett 65:1983-1985

5. Guderian A, Dechert G, Zeyer K, Schneider F (1996) Stochastic resonance in chemistry. 1 . The belousov-zhabotinsky reaction. J Phys Chem 100:4437-4441

6. Douglas JK, Wilkens L, Pantazelou E, Moss F (1993) Noise enhancement of information-transfer in crayfish mechanoreceptors by stochastic resonance. Nature 365:337-340

7. Mcnamara B, Wiesenfeld K, Roy R (1988) Observation of stochastic resonance in a ring laser. Phys Rev Lett 60:2626-2629

8. Mcnamara B, Wiesenfeld K (1989) Theory of stochastic resonance. Phys Rev A 39:4854-4869

9. Zhou T, Moss F, Jung P (1990) Escape-time distributions of a periodically modulated bistable system with noise. Phys Rev A 42:3161-3169

10. Jung P, Hänggi P (1991) Amplification of small signals via stochastic resonance. Phys Rev A 44:8032-8042

11. Dykman MI, Mannella R, McClintock PVE, Stocks NG (1990) Comment on "Stochastic Resonance in Bistable Systems". Phys Rev Lett 65:2606-2606

12. Dykman MI, Mannella R, McClintock PVE, Stocks NG (1992) Phase shifts in stochastic resonance. Phys Rev Lett 68:2985-2988

13. Dykman MI, Haken H, Hu Gang, Luchinsky DG, Mannella R, McClintock PVE, Ning CZ, Stein ND, Stocks NG (1993) Linear response theory in stochastic resonance. Phys Lett A 180:332-336

14. Gammaitoni L, Hänggi P, Jung P, Marchesoni F (1998) Stochastic resonance. Rev Mod Phys 70:223-287

15. Rosso OA, Masoller C (2009) Detecting and quantifying temporal correlations in stochastic resonance via information theory measures. Eur Phys J B 69:37-43

16. Rosso OA, Masoller C (2009) Detecting and quantifying stochastic and coherence resonances via information-theory complexity measurements. Phys Rev E 79:040106(R)1-4

17. Jia Y, Zheng X, Hu X, Li J (2001) Effects of colored noise on stochastic resonance in a bistable system subject to multiplicative and additive noise. Phys Rev E 63:031107

18. Zhang H, Xu W, Xu Y, Zhou B (2010) Delay induced teansitions in an asymmetry bistable system and stochastic resonance. Sci SinPhys Mech Astron 53:745-750

19. Sun Z, Yang X, Xu W (2012) Resonance dynamics evoked via noise recycling procedure. Phys Rev E 85:061125

20. López-Ruiz R, Mancini HL, Calbet X (1995) A statistical measure of complexity. Phys Lett A 209:321-326

21. Martin MT, Plastino A, Rosso OA (2003) Statistical complexity and disequilibrium. Phys Lett A 311:126-132

22. Lamberti PW, Martin MT, Plastino A, Rosso OA (2004) Intensive entropy non-triviality measure. Phys A 334:119-131

23. Rosso OA, Larrondo HA, Martin MT, Plastino A, Fuentes MA (2007) Distinguishing noise from chaos. Phys Rev Lett 99:154102

24. Martin MT, Plastino A, Rosso OA (2006) Generalized statistical complexity measures: geometrical and analytical properties. Phys A 369:439-462 
25. Rosso OA, Micco LD, Larrondo HA, Martín MT, Plastino A (2010) Generalized statistical complexity measure. Int J Bifurcat Chaos 20:775-785

26. Zunino L, Soriano MC, Fischer I, Rosso OA, Mirasso CR (2010) Permutation-information-theory approach to unveil delay dynamics from time-series analysis. Phys Rev E 82:046212

27. Hänggi P, Mroczkowski TJ, Moss F, McClintock PVE (1985) Bistability driven by colored noise: theory and experiment. Phys Rev A 32:695-698

28. Fox FR, Roy R (1987) Steady-state analysis of strongly colored multiplicative noise in a dye laser. Phys Rev A 35:1838-1842

29. Jung P, Hänggi P (1987) Dynamical systems: a unified colorednoise approximation. Phys Rev A 35:4464-4466
30. Jung P, Hänggi $\mathrm{P}$ (1988) Optical instabilities: new theories for colored-noise-driven laser instabilities. J Opt Soc Am B 5: 979-986

31. Cao L, Wu D (1995) Bistable kinetic model driven by correlated noises: unified colored-noise approximation. Phys Rev E 52: 3228-3231

32. Hu G (1994) Stochastic forces and nonlinear system. Shanghai Science and Technology Education Press, Shanghai

33. Bandt C, Pompe B (2002) Permutation entropy—a natural complexity measure for time series. Phys Rev Lett 88:174102 